\title{
Effect of many-body correlations on mesoscopic charge relaxation
}

\author{
Minchul Lee, ${ }^{1}$ Rosa López, ${ }^{2,3}$ Mahn-Soo Choi, ${ }^{4}$ Thibaut Jonckheere, ${ }^{5}$ and Thierry Martin ${ }^{5,6}$ \\ ${ }^{1}$ Department of Applied Physics, College of Applied Science, Kyung Hee University, Yongin 446-701, Korea \\ ${ }^{2}$ Departament de Física, Universitat de les Illes Balears, E-07122 Palma de Mallorca, Spain \\ ${ }^{3}$ Institut de Física Interdisciplinar i de Sistemes Complexos IFISC (CSIC-UIB), E-07122 Palma de Mallorca, Spain \\ ${ }^{4}$ Department of Physics, Korea University, Seoul 136-701, Korea \\ ${ }^{5}$ Centre de Physique Théorique, UMR6207, Case 907, Luminy, F-13288 Marseille Cedex 9, France \\ ${ }^{6}$ Université de la Méditerranée, F-13288 Marseille Cedex 9, France
}

(Received 1 April 2011; published 12 May 2011)

\begin{abstract}
We investigate nonperturbatively the charge relaxation resistance and quantum capacitance in a coherent $R C$ circuit in the strong-coupling regime. We find that the many-body correlations break the universality in the charge relaxation resistance: (i) The charge relaxation resistance has peaks at finite frequencies $\Gamma^{*} / \hbar$, where $\Gamma^{*}$ is an effective level broadening, and (ii) the zero-frequency resistance deviates from the universal value when the Zeeman splitting is comparable to $\Gamma^{*}$. This behavior becomes even more prominent in the Kondo regime. The observed features are ascribed to the generation of particle-hole excitations in the contacts accomplished by spin-flip processes in the dot.
\end{abstract}

DOI: 10.1103/PhysRevB.83.201304

PACS number(s): 73.63.Kv, 72.15.Qm, 73.23.-b

Introduction. For a macroscopic capacitor coupled to a reservoir [Fig. 1(a)], the low-frequency dynamical conductance is simple and determined solely by the geometrical capacitance and the resistance. As the capacitor scales down to nanometers size, the transport properties are significantly modified due to quantum coherence. First of all, the capacitance now depends strongly on the density of states (DOS) as well as the geometrical capacitance. More interestingly, the dynamical resistance becomes quantized, universal, and independent of the transmission through the mesoscopic conductor. ${ }^{1}$ The capacitance and resistance are called electrochemical capacitance and charge relaxation resistance, respectively, to be distinguished from the macroscopic counterparts.

So far, such a coherent $R C$ circuit has been described with various theoretical methods: the mean-field approach, ${ }^{2}$ the spin-polarized configurations, ${ }^{3,4}$ the perturbative treatment, ${ }^{5,6}$ and the semi-classical limit. ${ }^{7}$ However, a proper description of the many-body correlation effects is still missing. In this Rapid Communication, we attempt to fill this gap by treating the many-body interaction in a nonperturbative way.

We find that the many-body correlations break significantly the universality of the charge relaxation resistance and that the deviation is maximal at certain energy scale $\Gamma^{*}$, which we interpret as an effective level broadening. Namely, (i) the charge relaxation resistance has peaks at a finite frequency $\omega=\Gamma^{*} / \hbar$, and (ii) the zero-frequency charge relaxation resistance deviates significantly from the well-known universal value when the Zeeman splitting becomes comparable to $\Gamma^{*}$. Note that the peak structure cannot be explained in meanfield-like approaches, and that the interplay of the Zeeman splitting and the Coulomb interaction in the strong-coupling regime has not been studied before. Further, we find that the peak structure is most prominent in the Kondo regime, where $\Gamma^{*}$ turns out to be given by the Kondo temperature $T_{K}$. This is surprising because the Fermi liquid picture, which is widely used for the linear response conductance in the Kondo regime, fails to explain this behavior. Below we provide a clear interpretation of our findings in terms of the particle-hole (p-h) excitations accompanying a spinflip in the dot due to the strong Coulomb interaction, see Fig. 1(c).

The coherent $R C$ circuit is an interesting and promising electron analog of the on-demand single-photon source, which is an essential part of photon-based quantum information processing. The on-demand single-electron generation has been successfully demonstrated in a recent experiment, ${ }^{8}$ not long after the quantized charge relaxation resistance was observed experimentally. ${ }^{9}$ Encouraged by the two pioneering experiments $^{8,9}$ and motivated by the potential applications, there are a number of experimental studies of coherent $R C$ circuits. ${ }^{10-15}$

Model and Methods. We consider a nanoscale capacitor (quantum dot) coupled to a single reservoir. A weak timedependent external gate voltage $V(t)=V_{\text {ac }} \cos \omega t$ is applied on the quantum dot. In such a coherent $R C$ circuit, the ac transport is highly sensitive to the internal distribution of charges and potentials, which needs to be calculated in a self-consistently manner to ensure the gauge invariance and current conservation. To achieve this accurately, we use the numerical renormalization group (NRG) method, ${ }^{16,17}$ and calculate the charge relaxation resistance $R_{q}(\omega)$ and the electrochemical capacitance $C_{\mu}(\omega)$. Note that $C_{\mu}(\omega)$ is related to the geometrical capacitance $C$ and quantum capacitance $C_{q}(\omega)$ by $C_{\mu}=\left(C^{-1}+C_{q}^{-1}\right)^{-1}$.

The essential features of the system in the presence of correlations can be well captured in the Anderson model, where the dot is described by an interacting single level. The Hamiltonian $\mathcal{H}=\mathcal{H}_{\mathrm{L}}+\mathcal{H}_{\mathrm{D}}+\mathcal{H}_{\mathrm{T}}$ consists of the lead part

$$
\mathcal{H}_{\mathrm{L}}=\sum_{\mathbf{k} \mu}\left[\epsilon_{\mathbf{k}}+e V(t)\right] c_{\mathbf{k} \mu}^{\dagger} c_{\mathbf{k} \mu}
$$

the dot part

$$
\mathcal{H}_{\mathrm{D}}=\sum_{\mu}\left[\epsilon_{\mu}+e U(t)\right] d_{\mu}^{\dagger} d_{\mu}+2 E_{\mathrm{C}} d_{\uparrow}^{\dagger} d_{\downarrow}^{\dagger} d_{\downarrow} d_{\uparrow},
$$


(a)
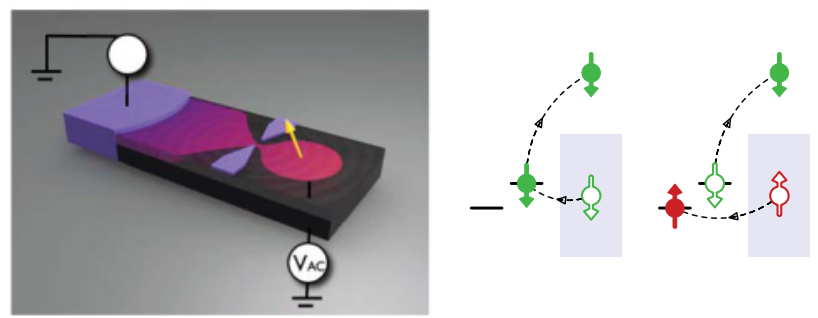

FIG. 1. (Color online) (left) Illustration of a quantum capacitor and (right) second-order tunneling processes that generate a single particle-hole pair in the conduction band (a) without and (b) with a spin flip in the dot. Filled and empty arrows in the contact indicates particles and holes, respectively. Here we assume $\Delta_{Z}>0$.

and the tunneling part

$$
\mathcal{H}_{\mathrm{T}}=\sum_{\mathbf{k} \mu}\left[t_{\mathbf{k}} d_{\mu}^{\dagger} c_{\mathbf{k} \mu}+\text { (H.c.) }\right]
$$

The operator $c_{\mathbf{k} \mu}$ describes the the noninteracting conduction electrons with energy $\epsilon_{\mathbf{k}}$ (measured with respect to the Fermi energy $\left.\epsilon_{F}=0\right)$ and spin $\mu$ in the single-channel reservoir and $d_{\mu}$ the interacting electron on the dot. $E_{\mathrm{C}}=e^{2} / 2 C$ is the Coulomb charging energy and $\epsilon_{\mu}=\epsilon_{d}-\mu \Delta_{Z} / 2$ are the dot levels with Zeeman splitting $\Delta_{Z}$ on the dot. $t_{\mathbf{k}}$ is the tunneling amplitude of electrons between the reservoir and the dot. For simplicity, we assume $t_{\mathbf{k}}=t$ and characterize the dot-lead hybridization by $\Gamma=\pi \rho_{0}|t|^{2}$ ( $\rho_{0}$ is the contact DOS at the Fermi energy).

The time-dependent voltage $V(t)$ induces the polarization charges $N_{U}(t)$ between the dot and the gate, which, in turn, leads to the time-dependent potential $U(t)=|e| N_{U}(t) / C$ inside the dot. Consequently, the applied voltage not only generates a current $I(t)$ between the lead and the dot, but also induces a dot-gate displacement current $I_{d}(t)=e\left(d N_{U} / d t\right)=$ $-C(d U / d t)$. Charge conservation requires $I(t)+I_{d}(t)=$ 0 . Assuming that the gate-invariant perturbation, $V(t)-$ $U(t)$, is sufficiently small, the linear response theory leads to the relation, $I(\omega)=g(\omega)[V(\omega)-U(\omega)]$, where $g(t)=$ $(i e / \hbar)\langle[\mathcal{I}(t), \mathcal{N}]\rangle \Theta(t)$ is the equilibrium correlation function between the occupation operator $\mathcal{N}=\sum_{\mu} d_{\mu}^{\dagger} d_{\mu}$ and the current operator $\mathcal{I}=e(d \mathcal{N} / d t)$. Note that the current-density correlation function $g(\omega)$ is directly related to the charge susceptibility $\chi_{c}(t)=-i\langle[\mathcal{N}(t), \mathcal{N}]\rangle \Theta(t)$, which is preferable for numerical computation, via the relation $g(\omega)=$ $i \omega\left(e^{2} / \hbar\right) \chi_{c}(\omega)$. Then, with the help of $I(\omega)=-I_{d}(\omega)=$ $-i \omega C U(\omega)$, the dot-lead impedance $Z(\omega)=V(\omega) / I(\omega)$, which is experimentally accessible, is given by $Z(\omega)=$ $1 /(-i \omega C)+1 / g(\omega)$. The relaxation resistance and the quantum capacitance are then expressed in terms of the charge susceptibility as

$$
\frac{R_{q}(\omega)}{h / e^{2}}=\operatorname{Re}\left[\frac{1}{2 \pi i \omega \chi_{c}(\omega)}\right], \quad \frac{e^{2} / h}{C_{q}(\omega)}=\operatorname{Im}\left[\frac{1}{2 \pi i \chi_{c}(\omega)}\right] .
$$

We first calculate the the imaginary part of the susceptibility using the NRG method, ${ }^{18}$ and then its real part via the Kramers-Kronig relation. Note that the NRG results for the finite-frequency linear response in the Kondo regime are known to be reliable as long as the time-dependent voltage


FIG. 2. (Color online) (a) Zero-frequency limits of $R_{q}$ and $C_{q}$ versus $\epsilon_{d}$. (b) Typical spectral structure of the real and imaginary parts of $\chi_{c}$. (c,d) $R_{q}(\omega)$ in the logarithmic scale. Here $\Gamma=0.04$, and $E_{\mathrm{C}}=0.5$.

$V(t)$ is weak enough. ${ }^{19}$ We focus on the zero-temperature case and use the contact bandwidth $D$ as the energy unit. We set $k_{B}=1$ hereafter unless specified.

No Zeeman splitting, $\Delta_{Z}=0$. Figure 2(a) shows the zerofrequency limit values of $R_{q}$ and $C_{q}$ for the spin-degenerate case. First, the zero-frequency limit of the relaxation resistance, $R_{q}(\omega \rightarrow 0)$ is always close to the universal value $h / 4 e^{2}$, regardless of values of $\epsilon_{d}$ and $E_{\mathrm{C}}$. This value can be interpreted as the composite resistance of two parallel resistors of resistance $h / 2 e^{2}$, the well-known universal resistance per channel. ${ }^{1,2,20}$ The NRG results show the quantization of charge relaxation even in the Kondo regime where many-body correlations are effective. The observed small deviations from the exact value $h / 4 e^{2}$, persisting even in the noninteracting case, are attributed to the finiteness of the contact bandwidth $D$, which introduces a frequency-dependent real part into the dot self-energy. ${ }^{21}$ The universal value can be restored by setting all the relevant energy scales to be much smaller than $D .^{22}$ Second, the quantum capacitance, $C_{q}$ exhibits two remarkable considerations: (i) at the degenerate points, $\epsilon_{d} \sim \epsilon_{F}$ and $\epsilon_{d}+2 E_{\mathrm{C}} \sim \epsilon_{F}, C_{q}$ shows two pronounced peaks [see Fig. 2(a)], which is consistent with the known understanding that $C_{q}$ is proportional to the $\operatorname{dot} \operatorname{DOS} \rho_{\mathrm{dot}}\left(\epsilon_{F}\right),{ }^{1,23}$ and (ii) $C_{q}$ remains quite small in the Kondo regime although the DOS at the Kondo resonant level pinned at the Fermi level achieves its maximum value. It implies that the Kondo resonant level, even though it can open a tunneling channel, is not a real level which can hold real charges and cannot contribute to the capacitance. Hence, in the presence of many-body correlations $C_{q}$ is not, always, directly related to the DOS.

The frequency dependence of $R_{q}(\omega)$ is shown in Fig. 2. One can clearly see that $R_{q}(\omega)$ exhibits two peaks at $\omega= \pm \Gamma^{*} / \hbar$, see Figs. 2(c) and 2(d) for two dot level positions, $\epsilon_{d}=$ -0.1 (fluctuating valence regime) and $\epsilon_{d}=-0.3$ (Kondo regime). One can interpret this structure in terms of the $\mathrm{p}-\mathrm{h}$ excitations and the relation in Eq. (4). $\operatorname{Im}\left[\chi_{c}(\omega)\right]$ reflects the coupling between the ground state and $\mathrm{p}-\mathrm{h}$ excitations via the dot-lead hybridization. Since the spectral density of multiple p-h excitations increases with energy, $\left|\operatorname{Im}\left[\chi_{c}\right]\right|$ would grow monotonically with $|\omega|$. However, a finite $D$ puts an upper limit to the energy for $\mathrm{p}$-h excitations $[|\omega| \gtrsim \mathcal{O}(D)]$ resulting in a 
nonmonotonic behavior for $\operatorname{Im}\left[\chi_{c}\right]$, see Fig. 2(b). Moreover, $\operatorname{Im}\left[\chi_{c}\right]$ has two kinks at $|\omega|=\min \left(\left|\epsilon_{d}\right|,\left|\epsilon_{d}+2 E_{\mathrm{C}}\right|\right)$ since beyond this frequency $\mathrm{p}-\mathrm{h}$ excitations accompanied with a charge excitation contributes to $\operatorname{Im}\left[\chi_{c}\right]$ as well. An interesting structure appears in $\operatorname{Im}\left[\chi_{c}\right]$ near $\omega=0$ [not seen in Fig. 2(b) due to logarithmically small energy scale]. Close to $\omega=0$, $\operatorname{Im}\left[\chi_{c}\right]$ depends linearly with $\omega$, mainly due to single $\mathrm{p}$-h excitations. However, $\operatorname{Im}\left[\chi_{c}\right]$ departs from the linearity when $\omega$ becomes of the order of the effective hybridization $\Gamma^{*}\left(=T_{K}\right.$ in the Kondo regime). Here the effective level broadening $\Gamma^{*}\left(T_{K}\right)$ is extracted from the width of the resonance close to(at) $\epsilon_{F}$ in $\rho_{\text {dot }}$. Besides, we found that the slope of $\operatorname{Im}\left[\chi_{c}(\omega)\right]$ is the largest at $\omega=\Gamma^{*} / \hbar$, and hence leading to the peak structure in $R_{q}(\omega)$ as seen in Figs. 2(c) and 2(d).

In the Kondo regime $R_{q}(\omega)$ becomes several orders of magnitude larger than the universal value, see Fig. 2(d). Note that such peak structure in $R_{q}(\omega)$ is absent in the noninteracting case and thus in the usual Fermi liquid picture of the Kondo effect. For a noninteracting system, $R_{q}(\omega)$ increases monotonically with increasing $|\omega|$, and the only characteristic energy scale is $\epsilon_{d}{ }^{24}$ Hence, the peaks seen in Figs. 2(c) and 2(d) are a genuine many-body effect.

Finite Zeeman splitting, $\Delta_{Z} \neq 0$. The spin-split case $\left(\Delta_{Z}>0\right)$ in the presence of external magnetic fields is illustrated in Fig. 3. In contrast to the spin-degenerate case, $R_{q}(\omega \rightarrow 0)$ is no longer fixed to the universal value. Interestingly, $R_{q}(\omega \rightarrow 0)$ versus $\Delta_{Z}$ exhibits a peak structure reaching values much larger than the quantized resistance observed in the spin-degenerate case: for example, $\left.R_{q}(\omega \rightarrow 0)\right|_{\max } \sim$ $100 \times h / 4 e^{2}$ for $\epsilon_{d}=-0.15$ (Kondo regime). Furthermore, the peak is exactly located at $\Delta_{Z}=\Gamma^{*}\left(T_{K}\right)$ for the fluctuating valence (Kondo) regime. The peak height increases as the the effective hybridization decreases so it is the highest in the Kondo regime. In the meanwhile, $C_{q}(\omega \rightarrow 0)$ remains rather constant, except at the resonant tunneling regime $\left(\epsilon_{d} \approx 0\right)$ where it displays a small peak, see Fig. 3(b). The evolution of the spectral distribution of $R_{q}(\omega)$ with $\Delta_{Z}$ is displayed in Fig. 3(c) for $\epsilon_{d}=-0.1$ (Kondo regime). As $\Delta_{Z}$ increases, the low-frequency part of $R_{q}(\omega)$ for $|\omega|<k_{B} T_{K} / \hbar$ keeps going up until $\Delta_{Z}$ reaches $k_{B} T_{K}$; the side peaks are merged into the central peak. With increasing $\Delta_{Z}$ further, the central peak diminishes gradually and, eventually, together with the side peaks located at $\omega= \pm k_{B} T_{K} / \hbar$, disappear completely. We have observed a similar transition of $R_{q}(\omega)$ with $\Delta_{Z}$ in the resonant tunneling regime $\left(\epsilon_{d}=-0.05,0\right)$ except that the variation of the central part is smaller.

To clarify the role of the Coulomb interaction, we compare the zero-frequency values of $R_{q}$ for different values of the Coulomb interaction in the resonant tunneling regime, see Fig. 3(d). In the noninteracting case $\left(E_{\mathrm{C}}=0\right)$, there is no peak at all, with $R_{q}(\omega \rightarrow 0)$ equal to $h / 4 e^{2}$. However, as soon as the charging energy $2 E_{\mathrm{C}}$ becomes comparable to $\Gamma^{*} \sim \Gamma$, a peak starts to rise up and manifests itself for $2 E_{\mathrm{C}} \gg \Gamma$. It implies that the existence of the peak structure observed in Fig. 3(d) definitely has its origin in the Coulomb interaction.

Discussion. Now we have two questions to be answered: (1) How can Coulomb interaction increase the relaxation resistance far beyond the universal value, $h / 4 e^{2}$ and (2) Why does it take place noticeably at $\omega= \pm \Gamma^{*}\left(T_{K}\right) / \hbar$ in the fluctuating valence (Kondo) regime for $\Delta_{Z}=0$ or at $\omega=0$ for
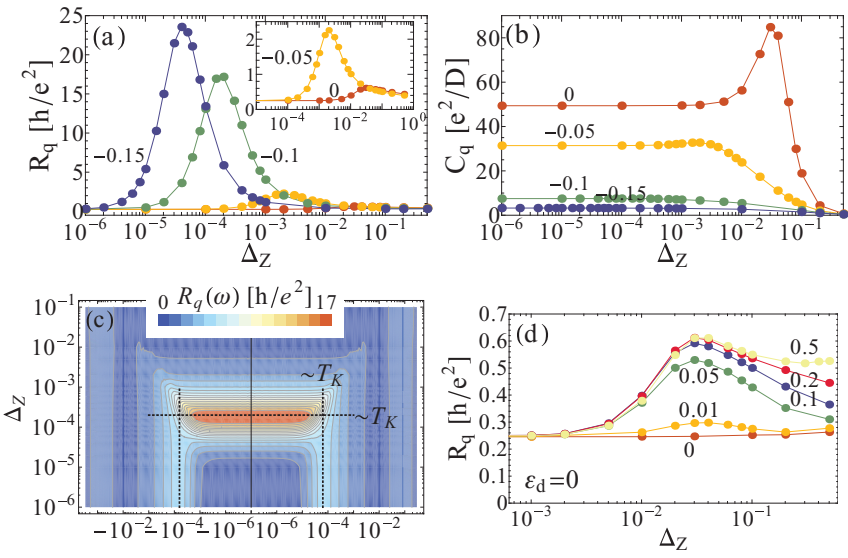

FIG. 3. (Color online) (a,b) $R_{q}(\omega \rightarrow 0)$ and $C_{q}(\omega \rightarrow 0)$ versus $\Delta_{Z}$ for annotated values of $\epsilon_{d}$ with $E_{\mathrm{C}}=0.2$. The enlarged view of $R_{q}(\omega \rightarrow 0)$ for large $\Delta_{Z}$ is shown in the inset. (c) Contour plot of $R_{q}$ as a function of $\omega$ and $\Delta_{Z}$ in the logarithmic scale in the Kondo regime $\left(\epsilon_{d}=-0.1\right)$. (d) $R_{q}(\omega \rightarrow 0)$ versus $\Delta_{Z}$ for $\epsilon_{d}=0$ and different values of $E_{\mathrm{C}}$ as annotated. Here $\Gamma=0.02$ is used.

$\Delta_{Z}=\Gamma^{*}\left(T_{K}\right)$ ? The charge relaxation resistance is attributed to $\mathrm{p}-\mathrm{h}$ pair generation in the conduction band as shown in Fig. 1. Such processes are put in action when the dot-lead tunneling is switched on. The tunneling, in turn, hybridizes dot and conduction band electrons, resulting in the lowering of the ground state by the effective binding energy $\Gamma^{*}\left(T_{K}\right.$ in the Kondo regime). It means that the $\mathrm{p}$-h generation starts when the energy supplied by the source is larger than $\Gamma^{*}$. This argument explains the observed peak in $R_{q}(\omega)$ at $\omega= \pm \Gamma^{*} / \hbar$ in the absence of the Zeeman splitting. In the presence of finite but small Zeeman splitting, the energy cost can be compensated by the Zeeman splitting. The energy of the $\mathrm{p}$-h pair excitation states shown in Fig. 1(c) are now lowered by $\Delta_{Z}$ compared to the states in Fig. 1(b), and when $\Delta_{Z} \approx \Gamma^{*}$ they become almost degenerate with the ground state, allowing $\mathrm{p}$-h pair generation with negligible energy cost. Hence, $R_{q}(\omega)$ exhibits a single peak at $\omega=0$ when $\Delta_{Z}=\Gamma^{*}$. This argument works solely when $\Delta_{Z} \lesssim \Gamma^{*}$ in which the ground state is not yet completely polarized and there exists a finite coupling among spin-down dot states and spin-up dot states accompanying with a $\mathrm{p}$-h pair generation in the reservoir, see Fig. 1 . The importance of the spin flip in the boosting of the relaxation resistance also explains why $R_{q}(\omega)$ can reach higher values in the Kondo regime. The Kondo ground state is built from spin fluctuations due to spin-flip scattering among the localized dot electron and the delocalized electrons in the reservoirs, thus spin-flip processes have large amplitudes in its wave function. Hence the processes as shown in Fig. 1(c) can happen more frequently, leading to a large $R_{q}$. Similarly, the answer for the first question is now ready. The spectral weight for the charge correlation function is proportional to $|\langle\alpha|\mathcal{N}| \mathrm{gs}\rangle|^{2}$, where gs and $\alpha$ represent the ground and the excited states. In the second-order perturbation theory, the weight corresponding to the processes in Fig. 1(c) is given by

$$
|\langle\alpha|\mathcal{N}| \mathrm{gs}\rangle|^{2}=t^{4}\left|\frac{2}{E_{\mu} E_{\bar{\mu}}}-\sum_{\mu} \frac{\mu}{\Delta_{Z}}\left(\frac{1}{E_{\mu}}+\frac{1}{\epsilon_{\mu}}\right)\right|^{2},
$$


in the $\omega \rightarrow 0$ limit with $E_{\mu}=2 E_{\mathrm{C}}+\epsilon_{\mu}$. Interestingly, this weight vanishes completely for $E_{\mathrm{C}}=0$ for any value of $\Delta_{Z}$. Thus, for the noninteracting case there exists no $\mathrm{p}-\mathrm{h}$ pair generation process accompanying spin flip in the dot, and no boosting of the relaxation resistance can happen. For finite values of $E_{\mathrm{C}}$, the weight is finite [see Eq. (5)] and for $E_{\mathrm{C}} \rightarrow \infty$, it becomes $t^{4} /\left(\epsilon_{\uparrow} \epsilon_{\downarrow}\right)^{2}$. This value can be substantial depending on the level position. Note that this analysis is not correct quantitatively because high-order events should be considerably involved in the observed phenomena. The observed boosting of $R_{q}$ at $\Delta_{Z} \sim \Gamma^{*}$ indicates that the perturbation in the dot-lead tunneling or $\Gamma$ is risky. A more general theoretical analysis that treats $\Delta_{Z}$ and $\Gamma$ on equal footing could provide more quantitatively reliable interpretation. Besides, this perturbative analysis does not work in the Kondo regime where the strong dot-lead coupling is important. One may want to study the Kondo regime by an effective single-particle Hamiltonian with a dot level at the Fermi energy with the effective hybridization $T_{K}$. However, this picture is only suitable in the Fermi-liquid regime in which p-h excitations accomplished by spin-flip events in the dot are not allowed. Besides, this effective model predicts an enhanced mesoscopic capacitance $C_{q}(\omega \rightarrow 0)$ due to the presence of the resonant level at the Fermi level. As noted before, however, the Kondo resonant level cannot contribute to the charging of real charges.

Conclusion. In closing, we have investigated the dynamics of a many-body quantum capacitor, focusing on the strong-coupling regime. We observe the breakdown of the Fermi-liquid features such as the quantized low-frequency relaxation resistance. We find that $R_{q}(\omega)$ shows peaks at $\omega= \pm \Gamma^{*}\left(T_{K}\right) / \hbar$ for $\Delta_{Z}=0$ and that $R(\omega \rightarrow 0)$ is enhanced over the quantized value when $\Delta_{Z}=\Gamma^{*}\left(T_{K}\right)$. It can be understood by the fact that a part of $R_{q}(\omega)$ is built by dot-lead tunneling events connecting $\mathrm{p}$-h excitations in the reservoirs with spin-flip processes in the dot. The boosted low-frequency (and high-frequency) relaxation resistance, which is much stronger in the Kondo regime, is expected to be experimentally observable by considering the current advances in $R C$-circuit experiments. $^{8,9}$

Acknowledgments. M. L. was supported by the NRF Grants (No. 2009-0069554 and No. 2010-0015416). R. L. was supported by MEC-Spain (Grant No. FIS2008-00781). T. M. and T. J. acknowledge support from ANR 2010 BLANC 0412 02. M.-S. C. was supported by the NRF Grants (No. 2009-0080453 and No. 2010-0025880).
${ }^{1}$ M. Büttiker, A. Prêtre, and H. Thomas, Phys. Rev. Lett. 70, 4114 (1993); M. Büttiker, A. Protre, and H. Thomas, Phys. Lett. A 180, 364 (1993).

${ }^{2}$ S. E. Nigg, R. López, and M. Büttiker, Phys. Rev. Lett. 97, 206804 (2006).

${ }^{3}$ K. Le Hur and G. Seelig, Phys. Rev. B 65, 165338 (2002).

${ }^{4}$ Y. Hamamoto et al., Phys. Rev. B 81, 153305 (2010).

${ }^{5}$ C. Mora and K. Le Hur, Nature Physics 6, 697 (2010).

${ }^{6}$ J. Splettstoesser et al., Phys. Rev. B 81, 165318 (2010).

${ }^{7}$ C. Petitjean et al., Phys. Rev. B 80, 115310 (2009).

${ }^{8} \mathrm{G}$. Fevè et al., Science 316, 1169 (2007).

${ }^{9}$ J. Gabelli et al., Science 313, 499 (2006).

${ }^{10}$ J. B. Pieper and J. C. Price, Phys. Rev. Lett. 72, 3586 (1994).

${ }^{11}$ L. P. Kouwenhoven et al., Phys. Rev. Lett. 73, 3443 (1994).

${ }^{12}$ M. Reznikov et al., Phys. Rev. Lett. 75, 3340 (1995).

${ }^{13}$ S. Verghese et al., Phys. Rev. B 52, 14834 (1995).

${ }^{14}$ R. J. Schoelkopf et al., Phys. Rev. Lett. 78, 3370 (1997).

${ }^{15}$ L.-H. Reydellet et al., Phys. Rev. Lett. 90, 176803 (2003).

${ }^{16}$ K. G. Wilson, Rev. Mod. Phys. 47, 773 (1975).

${ }^{17}$ H. R. Krishna-murthy, J. W. Wilkins, and K. G. Wilson, Phys. Rev. B 21, 1003 (1980); 21, 1044 (1980).
${ }^{18}$ For better accuracy, we adopt Hofstetter's algorithm (Ref. 25) accompanied with the improved $z$-averaging method (Ref. 26).

${ }^{19}$ M. Sindel et al., Phys. Rev. Lett. 94, 196602 (2005).

${ }^{20}$ Z. Ringel, Y. Imry, and O. Entin-Wohlman, Phys. Rev. B 78, 165304 (2008).

${ }^{21}$ Finite-width-band self-energy is given by $\operatorname{Re}[\Sigma(\omega)]=$ $-(\Gamma / 2 \pi) \ln (D-\hbar \omega) /(D+\hbar \omega)$. Its presence slightly violates the Fermi-liquid assumptions and, consequently, the KS relation is not exactly fulfilled so that the universal value is not recovered: Since $\operatorname{Re}[\Sigma(\omega)]$ increases with $\omega$, the deviations are larger as the resonant level becomes far from $\epsilon_{F}=0$.

${ }^{22}$ A. C. Hewson, J. Phys.: Cond. Mat. 18, 1815 (2006).

${ }^{23}$ M. Büttiker, J. Phys. Condens. Matter 5, 9361 (1993).

${ }^{24}$ The analytical expression of $R_{q}(\omega)$ in the wide band limit of the noninteracting system is given by Ref. 27: $R_{q}(\omega)=\left(h / e^{2}\right)\{[G(\omega)+$ $\left.(\hbar \omega / \Gamma) F(\omega)] /\left[G(\omega)^{2}+F(\omega)^{2}\right]\right\}$ with $G(\omega)=\ln \left\{\left[\left(\epsilon_{d}+\hbar \omega\right)^{2}+\right.\right.$ $\left.\left.\Gamma^{2}\right]\left[\left(\epsilon_{d}-\hbar \omega\right)^{2}+\Gamma^{2}\right] /\left[\epsilon_{d}^{2}+\Gamma^{2}\right]\right\}$ and $F(\omega)=2\left\{\tan ^{-1}\left[\Gamma /\left(\epsilon_{d}-\right.\right.\right.$ $\left.\hbar \omega)]-\tan ^{-1}\left[\Gamma /\left(\epsilon_{d}+\hbar \omega\right)\right]\right\}$.

${ }^{25}$ W. Hofstetter, Phys. Rev. Lett. 85, 1508 (2000).

${ }^{26}$ V. L. Campo and L. N. Oliveira, Phys. Rev. B 72, 104432 (2005); R. Zitko and T. Pruschke, ibid. 79, 085106 (2009).

${ }^{27}$ A.-P. Jauho, N. S. Wingreen, and Y. Meir, Phys. Rev. B 50, 5528 (1994). 